\title{
Circulating miRNAs reflect early myocardial injury and recovery after heart transplantation
}

Enshi Wang ${ }^{1,2}$, Yu Nie ${ }^{1}$, Qian Zhao ${ }^{1}$, Wei Wang ${ }^{2}$, Jie Huang ${ }^{3}$, Zhongkai Liao ${ }^{3}$, Hao Zhang ${ }^{1,2}$, Shengshou $\mathrm{Hu}^{1,2,4}$ and Zhe Zheng ${ }^{1,2,4^{*}}$

\begin{abstract}
Background: MicroRNAs (miRNAs) are short, single-stranded and non-coding RNAs, freely circulating in human plasma and correlating with vary pathologies. In this study, we monitored early myocardial injury and recovery after heart transplantation by detecting levels of circulating muscle-specific miR-133a, miR-133b and miR-208a.

Methods: 7 consecutive patients underwent heart transplantation in Fuwai hospital and 14 healthy controls were included in our study. Peripheral vein blood was drawn from patients on the day just after transplantation (day 0), the $1^{\text {st }}, 2^{\text {nd }}, 3^{\text {rd }}, 7^{\text {th }}$ and $14^{\text {th }}$ day after transplantation respectively. Serum from peripheral blood was obtained for cardiac troponin I (cTnl) measurement. Plasma was centrifuged from peripheral blood for measuring miR-133a, miR-133b and miR-208a by quantitative reverse transcription polymerase chain reaction (qRT-PCR). The plasma concentration of miRNAs were calculated by absolute quantification method. The sensitivity and specificity of circulating miRNAs were revealed by receiver operating characteristic curve (ROC) analysis. Correlations between miRNAs and cTnl / perioperative parameters were analyzed.
\end{abstract}

Results: Similar to cTnl, miR-133a, miR-133b and miR-208a all showed dynamic changes from high to low levels early after operation. The Sensitivity and specificity of miRNAs were: miR-133a (85.7\%,100\%), miR-208a (100\%,100\%), and miR-133b (90\%,100\%). Correlations between miRNAs and cTnl were statistically significant $(p<0.05)$, especially for miR-133b $\left(R^{2}=0.813, p<0.001\right)$. MiR-133b from Day 0-Day $2(r>0.98, p<0.01)$, and $c T n l$ from Day 1- Day 3 $(r>0.86, p<0.05)$ had strong correlations with bypass time, particularly parallel bypass time. Obviously, miR-133b had a better correlation than cTnl. Circulating miR-133b correlated well with parameters of heart function such as central venous pressure (CVP), pulmonary capillary wedge pressure (PCWP), cardiac output (CO) and inotrope support, while cTnl only correlated with 3 of the 4 parameters mentioned above. MiR-133b also had strong correlations with ventilation time $(r>0.99, p<0.001)$ and length of ICU stay $(r>0.92, p<0.05)$, both of which reflected the recovery after operation. The correlation coefficients of miR-133b were also higher than that of cTnl.

Conclusions: The dynamic change in circulating muscle-specific miRNAs, especially miR-133b can reflect early myocardial injury after heart transplantation. And miR-133b may have advantages over cTnl in forecasting graft dysfunction and recovery of patients after operation.

Keywords: Circulating miRNAs, Myocardial injury, Ischemia-reperfusion injury, Heart surgery, Heart transplantation

\footnotetext{
* Correspondence: zhengzhe@fuwai.com

${ }^{1}$ State Key Laboratory of Cardiovascular Disease, Fuwai Hospital, National Center for Cardiovascular Diseases, Chinese Academy of Medical Sciences and Peking Union Medical College, Beijing 100037, People's Republic of China

${ }^{2}$ Department of Cardiac Surgery, Research Center for Cardiac Regenerative Medicine, Fuwai Hospital, Chinese Academy of Medical Sciences and Peking Union Medical College, Beijing, China

Full list of author information is available at the end of the article
} 


\section{Background}

MicroRNAs (miRNAs) are single-stranded, non-coding RNAs with a length of 19-25 nucleotides, which negatively regulate gene expression by recognizing complementary messenger RNAs (mRNAs) and prohibiting their translation into functional protein [1]. The expression profile of miRNAs is found to be tissue-/cell-specific [2]. And aberrant expression of miRNAs can directly reflect disease status. Recently circulating cell-free miRNAs are found remarkably stable despite of repeated freezethawing, RNase digestion and other harsh conditions [3,4], partly because of the association with microvesicles or exosomes which are actively secreted or passively released into blood in physiological or pathological condition [5]; therefore, their potential as biomarker were evaluated in several cardiovascular diseases, such as coronary heart disease [6], acute myocardial infarction (AMI) [7-13], heart failure [14], and viral myocarditis [9]. MiR-133a, miR-133b and miR-208a, as cardiomyocyte-enriched miRNAs, become the common candidate miRNAs to study [7-13].

As the most effective therapy for end-stage heart disease, heart transplantation is widely used in many medical centers. The donor heart undergoes cold ischemia and ischemia-reperfusion injury during extracorporeal circulation, both of which induce necrosis and apoptosis of cardiomyocytes in donor heart and subsequent myocardial injury early after transplantation $[15,16]$. Review of the International Society for Heart and Lung Transplantation (ISHLT) Registry has shown that donor-related variables, particularly duration of myocardial ischemia, continue to emerge as an independent risk factor for early and late survival and cardiac allograft vasculopathy [17]. So it is very important to monitor the myocardial injury and give medical therapy at the early stage after transplantation. We hypothesized that the dynamic change of cardiomyocyte-enriched miRNAs in peripheral blood could reflect the myocardial injury and therefore predict recovery of heart function after heart transplantation. Thus we measured the circulating levels of miR-133a, miR-208a, and miR-133b in different time point after operation in the context of a sensitive myonecrosis marker, cardiac troponin I (cTnI), in 7 consecutive patients undergone heart transplantation.

\section{Methods}

\section{Patient selection}

From July $6^{\text {th }}$ to August $27^{\text {th }}, 2011,7$ consecutive patients underwent heart transplantation in Fuwai Hospital (Beijing, China) were included in our study (Table 1). 14 healthy people were also included as a control group. The protocol of this study was carried out according to the principles of the Declaration of Helsinki and approved by the Medical Ethics Committee of Cardiovascular
Table 1 Patient characteristics

\begin{tabular}{|c|c|}
\hline Number(n) & 7 \\
\hline Sex(male)(n) & 7 \\
\hline Age(years) & $46.71 \pm 12.31$ \\
\hline Body weight(kg) & $58.85 \pm 9.35$ \\
\hline \multicolumn{2}{|l|}{ Indication for cardiac transplantation(n) } \\
\hline Coronary artery disease & 1 \\
\hline Dilated cardiomyopathy & 5 \\
\hline Restrictive cardiomyopathy & 1 \\
\hline \multicolumn{2}{|l|}{ Perioperative parameters } \\
\hline Cold ischemia time of donor heart(min) & $284.29 \pm 66.92$ \\
\hline Bypass time(min) & $189.86 \pm 73.28$ \\
\hline Aortic clamping time(min) & $70.71 \pm 9.14$ \\
\hline $\mathrm{CVP}(\mathrm{mmHg})$ & $5.92 \pm 3.08$ \\
\hline PCWP(mmHg) & $9.16 \pm 2.59$ \\
\hline $\mathrm{CO}(\mathrm{L} / \mathrm{min})$ & $4.76 \pm 1.00$ \\
\hline Inotrope Score(micrograms /kg/min) & $7.72 \pm 3.48$ \\
\hline Ventilation time(min) & $38.43 \pm 24.64$ \\
\hline Length of ICU stay(days) & $3.49 \pm 2.46$ \\
\hline Use of ECMO after transplantation(n) & 2 \\
\hline \multicolumn{2}{|l|}{ Postoperative Echocardiography } \\
\hline Left atrium diameter(mm) & $41.29 \pm 7.76$ \\
\hline Left ventricular ejection fraction (\%) & $67.71 \pm 7.27$ \\
\hline Left ventricular end diastolic dimension (mm) & $43.57 \pm 4.47$ \\
\hline Interventricular septum(mm) & $9.43 \pm 1.40$ \\
\hline Right ventricular diameter(mm) & $21.57 \pm 5.88$ \\
\hline \multicolumn{2}{|l|}{ Immunosuppressive therapy(n) } \\
\hline Cyclosporine & 6 \\
\hline Tacrolimus & 1 \\
\hline Mycophenolate mofetil & 7 \\
\hline Azathioprine & 1 \\
\hline Sirolimus & 0 \\
\hline Prednisone & 7 \\
\hline
\end{tabular}

All value are presented as numbers or mean \pm SD. ICU Intensive care unit, $E C M O$ extracorporeal membrane oxygenation, CVP central venous pressure, PCWP pulmonary capillary wedge pressure, $C O$ cardiac output.

Institute and Fu Wai Hospital. Written informed consent was obtained from all the participants before enrolment.

\section{Blood sampling from patients}

$6 \mathrm{ml}$ venous blood samples were drawn from each patient immediately after transferred to ICU (day 0 ) and then at $6: 00 \mathrm{am}$ on the $1^{\text {st }}, 2^{\text {nd }}, 3^{\text {rd }}, 7^{\text {th }}$ and $14^{\text {th }}$ day after transplantation respectively. For serum separation, $3 \mathrm{ml}$ of each blood sample was put into a common test tube containing polyolefin resin (Greiner Bio-One, Frickenhausen, Germany) for at least $10 \mathrm{mi}-$ nutes at room temperature. Then the tube was centrifuged 
at $3000 \mathrm{~g}$ for 20 minutes to obtain serum. The left $3 \mathrm{ml}$ was put into a $\mathrm{K}_{2}$-EDTA-coated tubes (BD Biosciences, California, USA) and centrifuged at $1600 \mathrm{~g}$ for 10 minutes. The plasma was carefully transferred into a new RNA-free tube for second centrifugation at $16,000 \mathrm{~g}$ for $10 \mathrm{~min}$ to further eliminate cell debris. Then the plasma was aliquot into RNase-free tubes and stored at $-80^{\circ} \mathrm{C}$ before RNA extraction.

\section{RNA extraction}

Total RNA was extracted from 400 ul plasma, using the mirVana PARIS kit (Ambion, Warrington, United Kingdom) according to the modification of manufacturer's instructions (as detailed in ref [3]), and subsequently eluted in $30 \mathrm{ul}$ nuclease-free water. To date, several miRNAs such as miR-16 [18], or synthetic C. elegans miRNAs $[3,6,19]$ have been established and validated to normalize for the miRNAs content in different body fluid. However, for detection of miRNA in plasma, synthetic C. elegans miRNAs were broadly used $[3,6,19]$. So in our experiment cel-miR-39, cel-miR-54, and cel-miR-238 were chosen and spiked in the plasma samples after combining the sample with $2 \times$ Denaturing Solution (as a mixture of $25 \mathrm{fmol}$ of each oligonucleotide in a $5 \mathrm{ul}$ total volume) [3].

\section{Quantification of circulating miRNAs in plasma and cTnl in serum}

5 ul RNA was put into 15 ul reverse transcribed (RT) reaction to generate cDNA using the TaqMan miRNAs Reverse Transcription Kit and miRNAs-specific stemloop primers (Applied Biosystems, Foster City, USA). Quantitative reverse transcription polymerase chain reaction (qRT-PCR) was performed on ABI 7300 real-time PCR instrument (Applied Biosystems, Foster City, USA). The TaqMan 2× Universal PCR Master Mix (No AmpErase UNG) and TaqMan miRNAs Assay (Applied Biosystems, Foster City, USA) was used for quantification of miRNAs at $95^{\circ} \mathrm{C}$ for $10 \mathrm{~min}$, followed by $95^{\circ} \mathrm{C}$ for $15 \mathrm{~s}$ (40 cycles) and $60^{\circ} \mathrm{C}$ for $1 \mathrm{~min}$ ( 40 cycles). The $\mathrm{Ct}$ value was defined as the cycle number at which the fluorescence exceeded the threshold. In our experiment the detection limit of $\mathrm{Ct}$ value was defined as 40. All PCR results were duplicated to evaluate the miRNAs' expression level. cTnI levels in the serum were measured by the electrochemiluminescence method (Roche, Basel, Switzerland).

Generation of standard curves for absolute quantification of miRNAs and normalization of experimental data

Synthetic single-stranded RNA oligonucleotides miR133a, miR-208a, and miR-133b (miRBase Release v.19.0) were purchased from Shanghai GenePharma, China. Sequence information was provided in Table 2. Synthetic miRNAs were input into the RT reaction according to range of copies from reference $[3,20]$. The standard curves for miR133a, miR-208a, and miR-133b were plotted by $\mathrm{Ct}$ values versus copy number of the synthetic miRNAs as shown in Figure 1. Copies of endogenous miRNAs in plasma were then approximated according to their $\mathrm{Ct}$ values and the standard curve. A Normalization Factor was calculated by the $\mathrm{Ct}$ values of the three synthetic spiked-in C. elegans miRNAs $[3,20]$. Then the copies of a given miRNAs in each sample (calculated by the standard curves described earlier) were multiplied by the normalization factor corresponding to the sample to obtain a normalized copy number $[3,20]$.

\section{Cardiac function evaluation}

Swan-Ganz Catheter (Edwards Lifesciences, California, USA) was implanted through external jugular vein of patients before operation. Hemodynamic parameters such as central venous pressure (CVP), pulmonary capillary wedge pressure (PCWP) and cardiac output (CO) were measured on 0 day, the $1^{\text {st }}$ day, $2^{\text {nd }}$ day, or $3^{\text {rd }}$ day postoperation (within 48-72 hous after catherization). A score adapted from Wernovsky and his colleagues was used to quantify inotrope use for our patients [21]. Inotrope score is a reliable index of postoperative cardiac function; higher scores indicate poorer cardiac function. The score was calculated by obtaining the total amount of inotropic support the patients received at each sampling point (on 0 day, the $1^{\text {st }}$ day, $2^{\text {nd }}$ day, and $3^{\text {rd }}$ day postoperation) and then entering the data into the equation as follows: Inotrope score $=$ Dopamine + Dobutamine $+([$ Epinephrine + Norepinephrine + Phenylephrine $] \times 100)+$ Milrinone $\times 10$. Units of inotrope dosage

Table 2 Description of commercially purchased RNA oligonucleotides used in standard curve and spike-in experiments

\begin{tabular}{lll}
\hline miRNA & Manufacturer of synthetic miRNA & Synthetic miRNA (oligo) sequence \\
\hline cel-miR-39 & Shanghai GenePharma & UCACCGGGUGUAAAUCAGCUUG \\
cel-miR-54 & Shanghai GenePharma & UACCCGUAAUCUUCAUAAUCCGAG \\
cel-miR-238 & Shanghai GenePharma & UUUGUACUCCGAUGCCAUUCAGA \\
hsa-miR-133a & Shanghai GenePharma & UUUGGUCCCCUUCAACCAGCUG \\
hsa-miR-208a & Shanghai GenePharma & AUAAGACGAGCAAAAAGCUUGU \\
hsa-miR-133b & Shanghai GenePharma & UUUGGUCCCCUUCAACCAGCUA \\
\hline
\end{tabular}




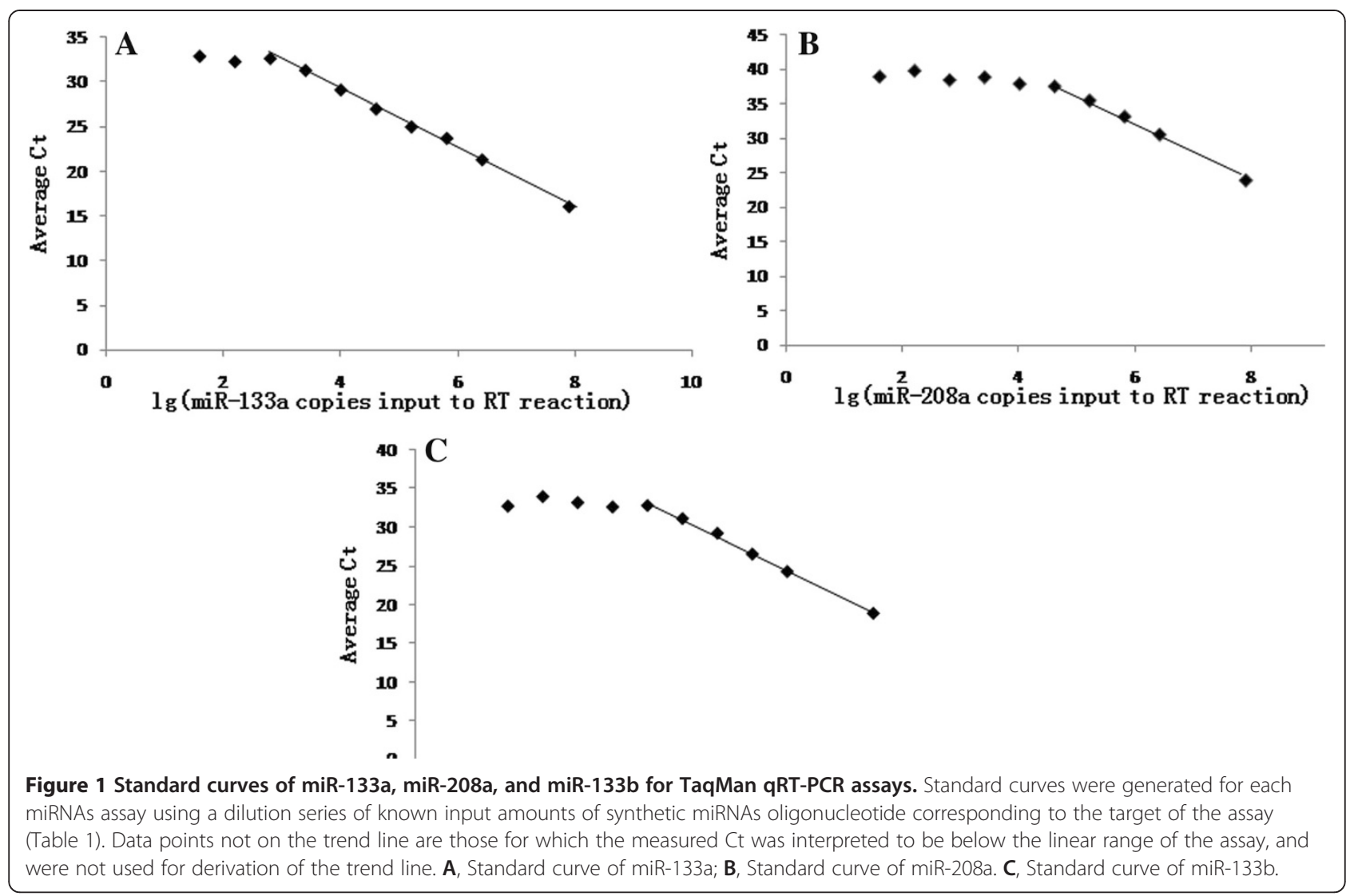

used in this equation were in micrograms per kilogram per minute.

\section{Statistical analyses}

All statistical data were presented as mean \pm standard deviation (SD) unless indicated otherwise. Pearson correlation analyses were used for miRNAs themselves or miRNAs versus cTnI and clinical parameters. Comparisons between 2 groups were performed with Student's t-tests for Gaussian data or Mann-Whitney tests for non-Gaussian data. For comparisons of more than 2 groups, one-way ANOVA was used, followed by post hoc testing using Bonferroni correction for more groups. The sensitivity and specificity of each miRNAs was calculated by receiver operating characteristic curve (ROC) analyses. All $\mathrm{p}$ values were two-sided and $\mathrm{p}$ values $<0.05$ were considered statistically significant. All analyses were performed using SPSS version 17.0.0 (SPSS Inc, Chicago, USA).

\section{Results}

Levels of circulating miR-133a, miR-208a and miR-133b elevated in patients after heart transplantation

The levels of circulating miR-133a, miR-208a, and miR133b were elevated to peak just after the transplantation (Day 0) (Figure 2A, 2C and 2E). MiR-133a increased to
$11675 \pm 3481$ copies/ul $(\mathrm{p}=0.001), \operatorname{miR}-208$ a to $5544 \pm$ 1757 copies/ul ( $<0.001)$, while miR-133b to $1173 \pm 227$ copies/ul $(\mathrm{p}<0.05)$ as compared to healthy controls. After that the expression levels of all three miRNAs in plasma declined gradually from Day 0 to Day 14 . However, miR-133a on Day 1 was $8198 \pm 1363$ copies/ul, which was still higher $(\mathrm{p}<0.05)$ than those in controls, so was miR208a (3966 \pm 1580 copies/ul, $\mathrm{p}=0.001)$. There seemed to be no difference for miRNAs in patients compared to controls after 2 days since heart transplantation except miR208a, which was still significant at Day $2(2852 \pm 550$ copies/ul, p < 0.05). Whereas, miR-133a, miR-208a, and miR-133b all showed a trend to decline gradually from Day 0 to Day 14, which was similar to serum cTnI (Figure 2B, 2D and 2F). Fold changes of miR-133a, miR208a and cTnI on Day 0 or Day 1 was significantly higher compared to Day 14 $(\mathrm{p}<0.05)$, although there were no significance on other time points. Of the 3 miRNA in plasma, miR-133a was the most abundant on Day 0, then followed by miR-208a and miR-133b. In all samples, concentration of miR-133a and miR-208a was statistically higher than that of miR-133b $(\mathrm{p}<0.05$ and $\mathrm{p}=0.001$ respectively), while there was no difference between miR133a and miR-208a. So we infer that circulating miR-133a and miR-208a are more enriched than miR-133b in plasma of patients after heart transplantation. 


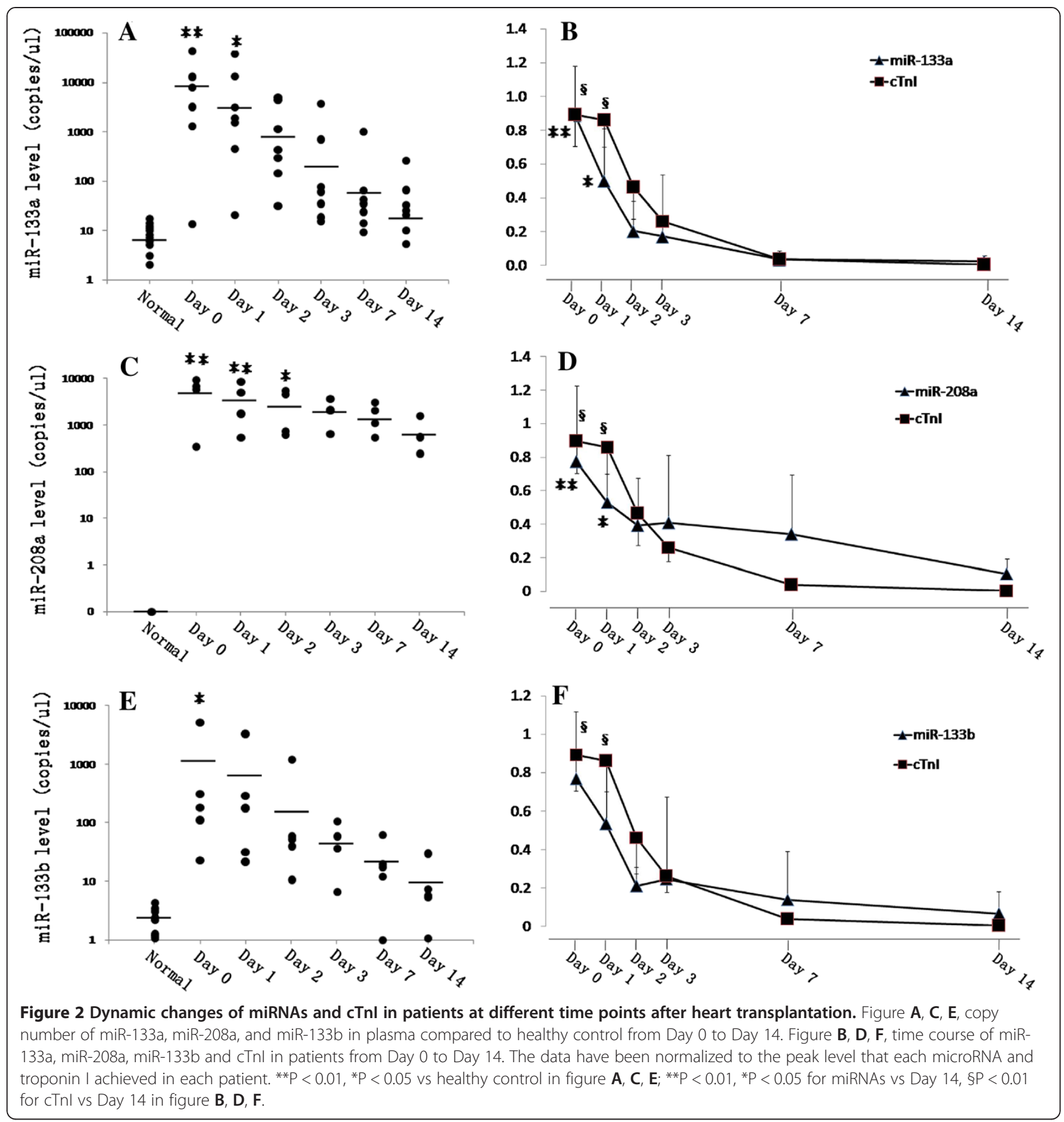

\section{Circulating miRNAs reflect myocardial injury effectively}

To evaluate whether circulating miRNAs can reflect the myocardial injury after heart transplantation, correlations between circulating miRNAs and cTnI were analyzed. We can see that all of these three circulating miRNAs in plasma correlated well with serum cTnI $(p<0.001)$ (Figure 3). MiR-133b had the stronger correlation with cTnI $\left(R^{2}=0.813, p<0.001\right.$; Figure $\left.3 E\right)$ compared with the correlations between miR-133a/miR-208a and cTnI $\left(\mathrm{R}^{2}=0.567, \mathrm{p}<0.001\right.$ and $\mathrm{R}^{2}=0.498, \mathrm{p}<0.001$ respectively;
Figure $3 \mathrm{~A}$ and $3 \mathrm{C}$ ). For correlations among miRNAs themselves, miR-133a, miR-208a and miR-133b correlated strongly with each other $(\mathrm{p}<0.001)$ (Figure 3B, 3D and $3 F)$. However, miR-208a which was expressed exclusively in heart, had a $100 \%$ sensitivity and specificity (Figure 4B), but it couldn't be detected in all patient samples (57.14\%), and not existed in healthy controls at all. Although miR133a was found in all samples and miR-133b could only be detected in $71.42 \%$ samples and $56.25 \%$ controls, miR$133 \mathrm{~b}$ had a better sensitivity than miR-133a (90\% 


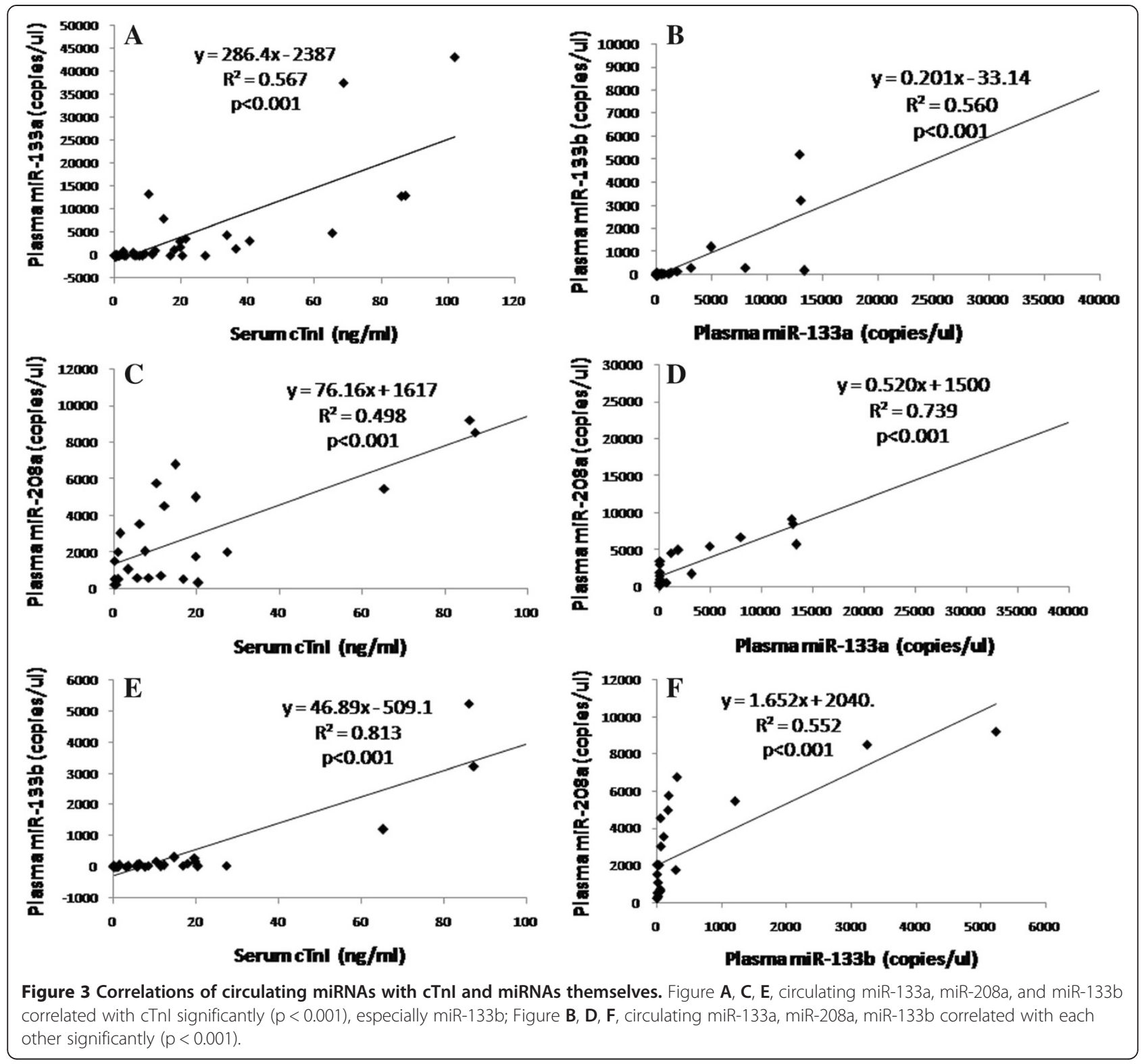

sensitivity and $100 \%$ specificity vs. $85.71 \%$ sensitivity and 100\% specificity; Figure 4A and 4C).

\section{Clinical correlations}

To evaluate the reason for rising of circulating miRNAs after transplantation and whether these miRNAs could predict the recent prognosis of patients, correlations of cirulating miRNAs at different time points with perioperative parameters were analyzed (Table 3 and Table 4). Neither circulating miRNAs nor cTnI had relationships with cold ischemia time of donor heart. However, miR-133b from Day 0-Day 2, cTnI from Day 1- Day 3, and miR-133a on Day 2 did have strong correlations with bypass time. The correlation coefficients of both miR-133a and miR-133b were over 0.98, which is higher than cTnI (Table 3). It seemed that length of bypass time contribute to elevations of miR-133b, cTnI and miR-133a. Next we divided bypass time into 2 parts: the aortic clamping time and parallel bypass time when there was a low level of reperfusion despite of persistent ischemia of heart. Interestingly, only miR-133a on Day 3 negatively correlated with the aortic clamping time, and miR-133b had a stronger association with parallel bypass time than cTnI from Day 0 to Day 2 (Table 3 ).

CVP, PCWP, CO, and inotrope score are indices of heart function. Higher CVP, PCWP, inotrope score and lower $\mathrm{CO}$ indicate worse dysfunction of heart. MiR133a, miR-208a, miR-133b, and cTnI had positive correlations with CVP and inotrope support (Table 4). However, only miR-133b and miR-208a had positive 

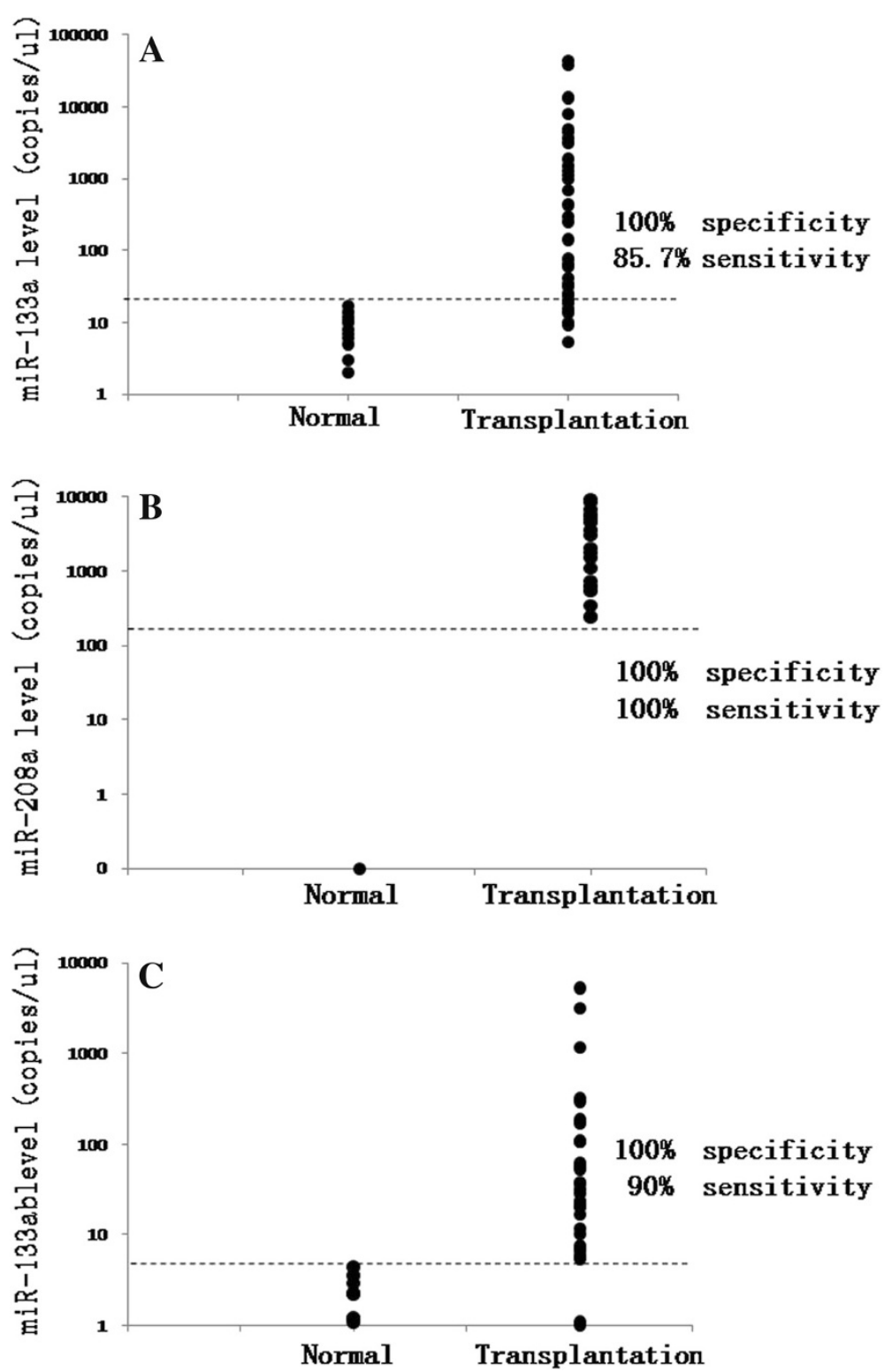

Figure 4 Sensitivity and specificity of circulating miRNAs. Figure A, Circulating miR-133a had $85.7 \%$ sensitivity and $100 \%$ specificity, whereas miR-208a had 100\% sensitivity and specificity in figure B. In figure C, for miR-133b, the sensitivity is $90 \%$, and specificity is $100 \%$.

correlations with PCWP; there were negative correlations between miR-133b/cTnI and CO (Table 4). So miR-133b was the only one that had the correlations with CVP, PCWP, CO, and inotrope score at the same time.

To evaluate recent prognosis, ventilation time and length of ICU stay were included. Ventilation time was strongly correlated with miR-133b from Day 0-Day 2, cTnI from Day 0-Day 3, and miR-133a on Day 2 ( $\mathrm{p}<0.05)$ (Table 3). The correlation coefficients of miR-133b were over 0.99 , still the highest of all. MiR-133b from Day 1Day 2, cTnI from Day 0-Day 3 showed correlationship with length of ICU stay $(\mathrm{p}<0.05)$ (Table 3$)$. The correlation coefficent of miR-133b at each time point was higher than that of cTnI.

\section{Discussion}

Recent studies on circulating cardiomyocyte-enriched miRNAs as biomarker for cardiovascular disease are growing rapidly because of their rapid release kinetics, cardiac selectivity, and stability in plasma [7-14,22,23]. In coronary artery disease, miR-133a, miR-208a, and miR-133b are considered cardio- or skeletal muscle specific and released into peripheral blood after AMI $[10,12,23]$. As we know, donor hearts for heart transplantation underwent cold ischemia (organ-preserving 
Table 3 Correlations between circulating miRNA and perioperative parameters

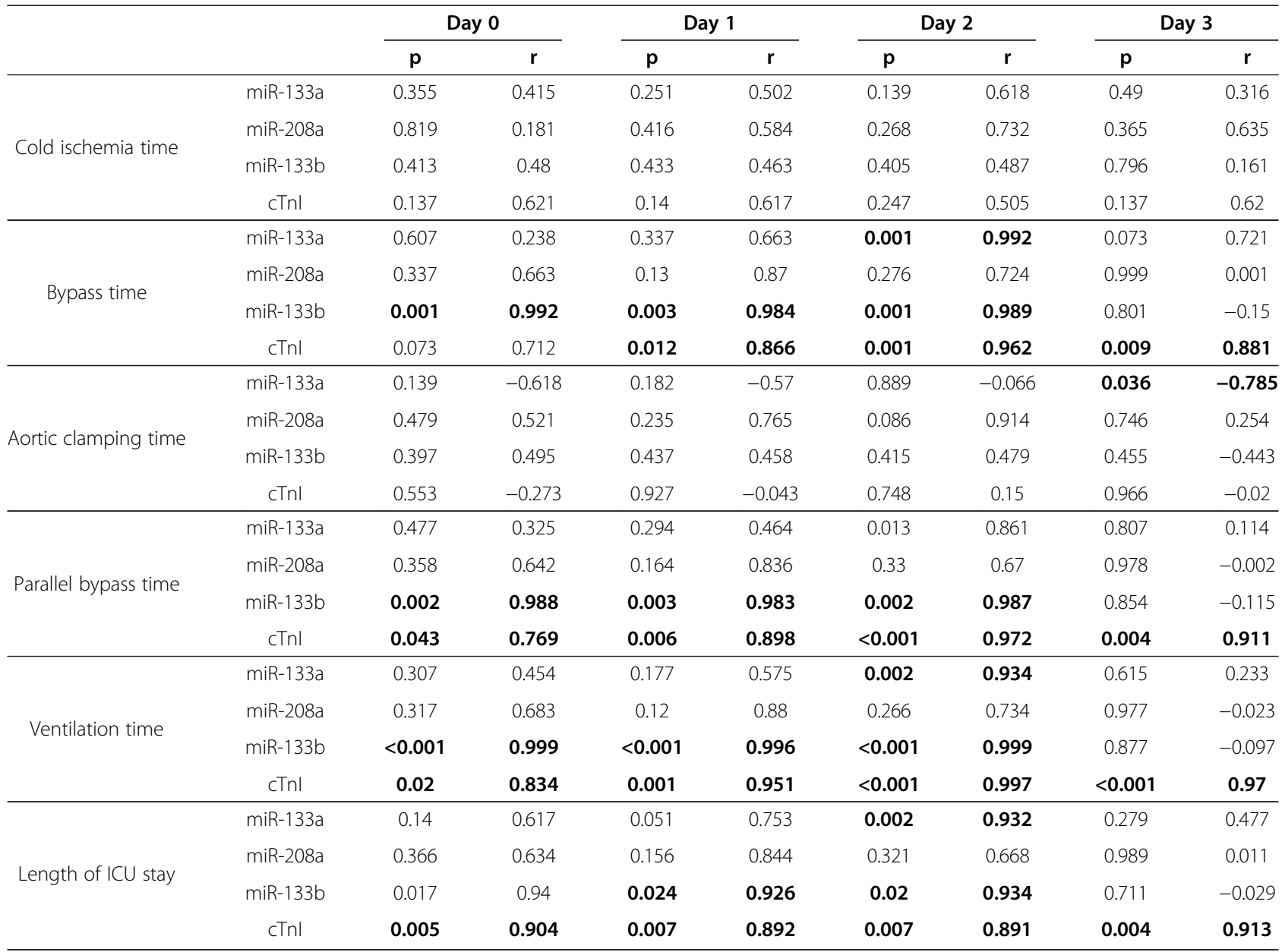

Statistically significant correlations are depicted in bold.

cold storage interval) and extracorporeal circulation before the heart completely restored function in recipients. Necrosis and apoptosis of cardiomyocytes occurred after ischemia in donor hearts [15,16,24-26]. Exosomes or microvesicles containing miRNAs will be released from cardiomyocytes into blood [27]. So miRNAs, enriched in heart like miR-133a, miR-208a and miR-133b, will be detected in plasma. To our knowledge, it is the first report to use circulating miR-133a, miR-208a, and miR133b monitoring donor heart injury after transplantation. Several new important observations are found in our study: 1) levels of circulating miR-133a, miR-208a and miR-133b are elevated in patients after heart transplantation and show a dynamic change; 2) circulating miR-133a, miR-208a, and miR-133b could reflect the heart injury after transplantation effectively; 3) circulating miRNAs are suggested to be a good predictive parameter for graft dysfunction and recent prognosis of patients after operation, especially miR-133b, which might be better than cTnI.

In our study, the levels of circulating miR-133a, miR208a and miR-133b were peaked just after heart

Table 4 Correlations between circulating miRNA and parameters of cardiac function

\begin{tabular}{|c|c|c|c|c|c|c|c|c|}
\hline & \multicolumn{2}{|c|}{ CVP } & \multicolumn{2}{|c|}{ PCWP } & \multicolumn{2}{|c|}{$\mathrm{CO}$} & \multicolumn{2}{|c|}{ Inotrope score } \\
\hline & $p$ & $\mathbf{r}$ & $p$ & $\mathbf{r}$ & $p$ & $\mathbf{r}$ & $p$ & $r$ \\
\hline miR-133a & 0.001 & 0.656 & 0.719 & 0.098 & 0.450 & -0.290 & 0.001 & 0.605 \\
\hline miR-208a & 0.011 & 0.677 & $<0.001$ & 0.962 & 0.401 & -0.599 & 0.018 & 0.581 \\
\hline miR-133b & 0.002 & 0.692 & 0.017 & 0.730 & 0.049 & -0.880 & $<0.001$ & 0.861 \\
\hline cTnl & $<0.001$ & 0.728 & 0.117 & 0.407 & 0.043 & -0.681 & $<0.001$ & 0.756 \\
\hline
\end{tabular}


transplantation, which was similar to cTnI. The time point of Day 0 was at the moment patients just transferred into intensive care unit (ICU) from operating room after heart transplantation. So all of our sampling time points were after operation, not during the operation. Maybe that's the reason why the cTnI and circulating miRNAs peaked at the same time point, not like reference before that circulating miRNAs peaked in 1-4 hours which was earlier than cTnI after AMI $[10,13]$. Despite of it, 3 of 7 patients did show a peak of cTnI at Day 1 later than miRNAs that leads to a general mild slope from Day 0 to Day 1 . All of these three miRNAs showed sharp slopes (Figure 2), which was similar to dynamic change of miRNAs in ST-segment elevation myocardial infarction (STEMI) patients [10,28]. Overall of it, there was no difference between trend of cTnI and miRNAs $(p>0.05)$. Like cTnI, circulating miR-133a, miR-208a and miR-133b showed gradually declined trend as time passed, which was similar to the trend of implanted heart recovering from injury after cold ischemia and extracorporeal circulation insults, then restoring function as a normal heart.

Studies before showed circulating miRNAs correlated with cTnI or cardiac troponin $\mathrm{T}$ (cTnT) at varying degrees $[9,19,29]$. In our study, we found three circulating miRNAs were correlated with cTnI well, especially miR$133 \mathrm{~b}$, which was strongly associated with $\mathrm{c} \operatorname{TnI}\left(\mathrm{R}^{2}=0.813\right.$, $\mathrm{p}<0.001$ ) (Figure $3 \mathrm{E}$ ). Since cTnI is widely used as a myonecrosis marker for diagnosis of AMI [30], we would like to infer that circulating miRNAs could reflect the myocardial injury of donor heart after transplantation. Heart-specific miR-208a got a 100\% sensitivity and specificity (Figure 4B), but it could only be detected in 57.14\% samples. This result was similar to the report in which miR-208a was expressed at low levels in three of nine AMI patients and undetectable in plasma samples from healthy human [10]. The low detected rate limits its application in the future. MiR-133a could be measured in all patients, but its sensitivity is only $85.71 \%$ (Figure 4A), while miR-133b with a $90 \%$ sensitivity and $100 \%$ specificity seemed to be the compromise (Figure 4C).

As we know, extrocoporeal circualtion could lead to heart injury [26]. The present study showed bypass time was strongly associated with miR-133b on Day 0 $(\mathrm{r}=0.992, \mathrm{p}=0.001)$, Day $1(\mathrm{r}=0.984, \mathrm{p}=0.003)$, and Day $2(\mathrm{r}=0.989, \mathrm{p}=0.001)$ (Table 3$)$. This is better than $\mathrm{cTnI}$ with later time points and lower values. The parallel bypass time that stands for reperfusion period plays a major role in elevation of miR-133b, or in other words, heart injury. Experimental studies have indeed shown that reperfusion itself initiates a process by which part of the salvageable myocardium fails to be rescued [31,32]. Oxygen depletion during ischemia inhibits mitochondrial function, which leads to a surge of reactive oxygen species (ROS) during reperfusion $[32,33]$. ROS are toxic to the cell and could lead to cell death. Then more miR-133b were released from dead cardiomyocytes into plasma. That may explain the high correlations of miR-133b concentration with parallel bypass time. Although miR-133b is present in sekeletal muscle and bypass causes skeletal muscle ischemia and reperfusion, previous study confirmed circulating miR133b didn't elevate, but decreased within $24 \mathrm{~h}$ following acute ischaemia of skeletal muscle and then back to control level [10]. So Elevation of miR-133b from skeletal muscle sources could be excluded in our study. The present study suggests miR-133b play a better role than $\mathrm{cTnI}$ in reflecting myocardial reperfusion injury (Table 3).

It is well-known that elevated cTnI or cTnT from donor heart is associated well with donor heart dysfunction or graft failure on hemodynamic, echocardiography or inotrope support [34-37]. In our study, cTnI levels in recipients were correlated well with CVP, $\mathrm{CO}$ and inotrope support at different time points after heart transplantation (Table 4). The tendency was in accordance with previous studies [34-37], although the cTnI data in our experiment were from recipients after transplantation but not from donors. We also found these 3 circulating miRNA were associated with hemodynamic indices and inotrope support. Among them, miR-133b was still the best one to predict graft dysfunction, because miR-133b was the only one that had correlations with CVP, PCWP, CO, and inotrope support. The other 2 miRNAs and cTnI had correlations with only 2 or 3 of the 4 parameters (Table 4). Evidence from our experiment suggested that circulating miR-133b predict graft dysfunction better than cTnI.

MiRNAs like miR-133a, miR-208b can provide prognostic information in acute coronary syndrome [12,23]. In this study, we found miR-133b had the best correlation with ventilation time and length of ICU stay (Table 3). The length of ventilation and ICU stay reflects the recovery of patients after operation. So circulating miR-133b not only plays an important role in monitoring heart injury but also acts as a prognostic factor for patients' recovery. Recent functional studies indicated that miR-133 had effects in the regulation of stressinduced myocyte survival with an antiapoptotic role [38]. This might point towards a cardio-protective role for these miRNAs in heart transplantation, which needs to be addressed thoroughly in future studies.

Some limitations are avaliable in the current study. First of all, the sample size is small. A large number of patients undergoing cardiac surgery will be included in our following study. Secondly, We didn't collect the blood samples during the operation, which may partly explain the peaks of miRNAs were not earlier than that 
of cTnI. Thirdly, miRNA measurement is time-consuming at present, limiting the clinical use of the results. Technology needs to be developed to allow quick bedside test and broad use of miRNAs as biomarkers.

\section{Conclusions}

The present study suggests circulating miRNAs, especially miR-133b be a new sensitive marker for monitoring and forcasting myocardial injury and recovery after heart transplantation. Our investigation also uncovers a new field for biomarker studies in cardiovascular surgery, such as coronary artery bypass grafting (CABG), cardiac valve replacement, or congenital heart disease (CHD) surgery, most of which undergo extracorporeal circulation.

\begin{abstract}
Abbreviations
CTnl: Cardiac troponin l; miRNAs: microRNAs; mRNAs: messenger RNAs; AMI: Acute myocardial infarction; qRT-PCR: quantitative reverse transcription polymerase chain reaction; SD: Standard deviation; ROC: Receiver operating characteristic curve; ROS: Reactive oxygen species; CABG: Coronary artery bypass surgery; CHD: Congenital heart disease; ISHLT: International Society for Heart and Lung Transplantation; STEMI: ST-segment elevation myocardial infarction; AMI: Acute myocardial infarction; CVP: Central venous pressure; PCWP: Pulmonary capillary wedge pressure; CO: Cardiac output.
\end{abstract}

\section{Competing interests}

The authors declare that they have no competing interests.

\section{Authors' contributions}

EW, ZZ and SH carried out the conception and design. EW carried out the sample collection, experiment, data analysis, and interpretation. YN participated in experiment. ZL and JH participated in the sample collection. WW participated in the interpretation. EW carried out writing the article, $\mathrm{SH}$, $\mathrm{ZZ}$, and $\mathrm{HZ}$ participated in the critical revision of the article and the statistical analysis. QZ participated in data analysis and language correction. All authors read and approved the final manuscript.

\section{Acknowledgement}

The current study was supported by Chinese government "863" program "Key technology of heart transplantation and immune regulation". No: 2012AA021009.

\begin{abstract}
Author details
${ }^{1}$ State Key Laboratory of Cardiovascular Disease, Fuwai Hospital, National Center for Cardiovascular Diseases, Chinese Academy of Medical Sciences and Peking Union Medical College, Beijing 100037, People's Republic of China. ${ }^{2}$ Department of Cardiac Surgery, Research Center for Cardiac Regenerative Medicine, Fuwai Hospital, Chinese Academy of Medical Sciences and Peking Union Medical College, Beijing, China. ${ }^{3}$ Department of Heart Transplantation, Fuwai Hospital, Chinese Academy of Medical Sciences and Peking Union Medical College, Beijing, China. ${ }^{4}$ Department of Cardiac Surgery, State Key Laboratory of Cardiovascular Disease, Fuwai Hospital, Chinese Academy of Medical Sciences, Xicheng District, Beijing 100037, China.
\end{abstract}

Received: 20 February 2013 Accepted: 26 June 2013

Published: 1 July 2013

\section{References}

1. Bartel DP: MicroRNAs: genomics, biogenesis, mechanism, and function. Cell 2004, 116:281-297.

2. Kloosterman WP, Plasterk $\mathrm{RH}$ : The diverse functions of microRNAs in animal development and disease. Dev Cell 2006, 11:441-450.

3. Mitchell PS, Parkin RK, Kroh EM, Fritz BR, Wyman SK, Pogosova-Agadjanyan EL, Peterson A, Noteboom J, O'Briant KC, Allen A, et al: Circulating
microRNAs as stable blood-based markers for cancer detection. Proc Natl Acad Sci USA 2008, 105:10513-10518

4. Chen X, Ba Y, Ma L, Cai X, Yin Y, Wang K, Guo J, Zhang Y, Chen J, Guo X, et al: Characterization of microRNAs in serum: a novel class of biomarkers for diagnosis of cancer and other diseases. Cell Res 2008, 18:997-1006.

5. Reid G, Kirschner MB, Van Zandwijk N: Circulating microRNAs: Association with disease and potential use as biomarkers. Crit Rev Oncol Hematol 2011, 80:193-208.

6. Fichtlscherer S, De Rosa S, Fox H, Schwietz T, Fischer A, Liebetrau C, Weber M, Hamm CW, Roxe T, Muller-Ardogan M, et al: Circulating microRNAs in patients with coronary artery disease. Circ Res 2010, 107:677-684.

7. Adachi T, Nakanishi M, Otsuka Y, Nishimura K, Hirokawa G, Goto Y, Nonogi H, Iwai N: Plasma microRNA 499 as a biomarker of acute myocardial infarction. Clin Chem 2010, 56:1183-1185.

8. Ai J, Zhang R, Li Y, Pu J, Lu Y, Jiao J, Li K, Yu B, Li Z, Wang R, et al: Circulating microRNA-1 as a potential novel biomarker for acute myocardial infarction. Biochem Biophys Res Commun 2010, 391:73-77.

9. Corsten MF, Dennert R, Jochems S, Kuznetsova T, Devaux Y, Hofstra L, Wagner DR, Staessen JA, Heymans S, Schroen B: Circulating MicroRNA-208b and MicroRNA-499 reflect myocardial damage in cardiovascular disease. Circ Cardiovasc Genet 2010, 3:499-506.

10. D'Alessandra Y, Devanna P, Limana F, Straino S, Di Carlo A, Brambilla PG, Rubino M, Carena MC, Spazzafumo L, De Simone M, et al: Circulating microRNAs are new and sensitive biomarkers of myocardial infarction. Eur Heart J 2010, 31:2765-2773.

11. Olivieri F, Antonicelli R, Lorenzi M, D'Alessandra Y, Lazzarini R, Santini G, Spazzafumo L, Lisa R, La Sala L, Galeazzi R, et al: Diagnostic potential of circulating miR-499-5p in elderly patients with acute non ST-elevation myocardial infarction. Int J Cardiol 2013, 167:531-536.

12. Widera C, Gupta SK, Lorenzen JM, Bang C, Bauersachs J, Bethmann K, Kempf T, Wollert KC, Thum T: Diagnostic and prognostic impact of six circulating microRNAs in acute coronary syndrome. J Mol Cell Cardiol 2011, 51:872-875

13. Wang GK, Zhu JQ, Zhang JT, Li Q, Li Y, He J, Qin YW, Jing Q: Circulating microRNA: a novel potential biomarker for early diagnosis of acute myocardial infarction in humans. Eur Heart J 2010, 31:659-666.

14. Tijsen AJ, Creemers EE, Moerland PD, De Windt LJ, van der Wal AC, Kok WE, Pinto YM: MiR423-5p as a circulating biomarker for heart failure. Circ Res 2010, 106:1035-1039.

15. Chiong M, Wang ZV, Pedrozo Z, Cao DJ, Troncoso R, Ibacache M, Criollo A, Nemchenko A, Hill JA, Lavandero S: Cardiomyocyte death: mechanisms and translational implications. Cell Death Dis 2011, 2:e244.

16. Li S, Guan Q, Chen Z, Gleave ME, Nguan CY, Du C: Reduction of cold ischemia-reperfusion injury by graft-expressing clusterin in heart transplantation. J Heart Lung Transplant 2011, 30:819-826.

17. Taylor DO, Stehlik J, Edwards LB, Aurora P, Christie JD, Dobbels F, Kirk R, Kucheryavaya AY, Rahmel AO, Hertz MI: Registry of the International Society for Heart and Lung Transplantation: Twenty-sixth Official Adult Heart Transplant Report-2009. J Heart Lung Transplant 2009, 28:1007-1022.

18. Cui L, Zhang X, Ye G, Zheng T, Song H, Deng H, Xiao B, Xia T, Yu X, Le Y, Guo J: Gastric juice MicroRNAs as potential biomarkers for the screening of gastric cancer. Cancer 2013, 119:1618-1626

19. De Rosa S, Fichtlscherer S, Lehmann R, Assmus B, Dimmeler S, Zeiher AM: Transcoronary concentration gradients of circulating microRNAs. Circulation 2011, 124:1936-1944.

20. Kroh EM, Parkin RK, Mitchell PS, Tewari M: Analysis of circulating microRNA biomarkers in plasma and serum using quantitative reverse transcription-PCR (qRT-PCR). Methods 2010, 50:298-301.

21. Wernovsky G, Wypij D, Jonas RA, Mayer JE Jr, Hanley FL, Hickey PR, Walsh AZ, Chang AC, Castaneda AR, Newburger JW, et al: Postoperative course and hemodynamic profile after the arterial switch operation in neonates and infants. A comparison of low-flow cardiopulmonary bypass and circulatory arrest. Circulation 1995, 92:2226-2235.

22. Ji X, Takahashi R, Hiura Y, Hirokawa G, Fukushima Y, Iwai N: Plasma miR-208 as a biomarker of myocardial injury. Clin Chem 2009, 55:1944-1949.

23. Eitel I, Adams V, Dieterich P, Fuernau G, De Waha S, Desch S, Schuler G, Thiele $\mathrm{H}$ : Relation of circulating MicroRNA-133a concentrations with myocardial damage and clinical prognosis in ST-elevation myocardial infarction. Am Heart J 2012, 164:706-714.

24. De Groot-Kruseman HA, Baan CC, Zondervan PE, De Weger RA, Niesters HG, Balk AH, Weimar W: Apoptotic death of infiltrating cells in human cardiac 
allografts is regulated by IL-2, FASL, and FLIP. Transplant Proc 2004, 36:3143-3148.

25. Khoynezhad A, Jalali Z, Tortolani AJ: Apoptosis: pathophysiology and therapeutic implications for the cardiac surgeon. Ann Thorac Surg 2004, 78:1109-1118.

26. Kovacevic M, Simic O, Jonjic N, Stifter S: Apoptosis and cardiopulmonary bypass. J Card Surg 2007, 22:129-134.

27. Cocucci E, Racchetti G, Meldolesi J: Shedding microvesicles: artefacts no more. Trends Cell Biol 2009, 19:43-51.

28. Gidlof O, Andersson P, van der Pals J, Gotberg M, Erlinge D: Cardiospecific microRNA plasma levels correlate with troponin and cardiac function in patients with ST elevation myocardial infarction, are selectively dependent on renal elimination, and can be detected in urine samples. Cardiology 2011, 118:217-226.

29. Kuwabara Y, Ono K, Horie T, Nishi H, Nagao K, Kinoshita M, Watanabe S, Baba O, Kojima Y, Shizuta S, et al: Increased microRNA-1 and microRNA133a levels in serum of patients with cardiovascular disease indicate myocardial damage. Circ Cardiovasc Genet 2011, 4:446-454.

30. Morrow DA, Cannon CP, Jesse RL, Newby LK, Ravkilde J, Storrow AB, Wu AH, Christenson RH: National Academy of Clinical Biochemistry Laboratory Medicine Practice Guidelines: Clinical characteristics and utilization of biochemical markers in acute coronary syndromes. Circulation 2007, 115:e356-e375.

31. Hausenloy DJ, Yellon DM: Time to take myocardial reperfusion injury seriously. N Engl J Med 2008, 359:518-520.

32. Yellon DM, Hausenloy DJ: Myocardial reperfusion injury. N Engl J Med 2007, 357:1121-1135.

33. Becker LB: New concepts in reactive oxygen species and cardiovascular reperfusion physiology. Cardiovasc Res 2004, 61:461-470.

34. Venkateswaran RV, Ganesh JS, Thekkudan J, Steeds R, Wilson IC, Mascaro J, Thompson R, Bonser RS: Donor cardiac troponin-l: a biochemical surrogate of heart function. Eur I Cardiothorac Surg 2009, 36:286-292. discussion 292.

35. Grant JW, Canter CE, Spray TL, Landt Y, Saffitz JE, Ladenson JH, Jaffe AS: Elevated donor cardiac troponin I. A marker of acute graft failure in infant heart recipients. Circulation 1994, 90:2618-2621.

36. Anderson JR, Hossein-Nia M, Brown P, Holt DW, Murday A: Donor cardiac troponin-T predicts subsequent inotrope requirements following cardiac transplantation. Transplantation 1994, 58:1056-1057.

37. Potapov EV, Ivanitskaia EA, Loebe M, Mockel M, Muller C, Sodian R, Meyer R, Hetzer R: Value of cardiac troponin I and T for selection of heart donors and as predictors of early graft failure. Transplantation 2001 71:1394-1400.

38. Xu C, Lu Y, Pan Z, Chu W, Luo X, Lin H, Xiao J, Shan H, Wang Z, Yang B: The muscle-specific microRNAs miR-1 and miR-133 produce opposing effects on apoptosis by targeting HSP60, HSP70 and caspase-9 in cardiomyocytes. J Cell Sci 2007, 120:3045-3052.

doi:10.1186/1749-8090-8-165

Cite this article as: Wang et al:: Circulating miRNAs reflect early

myocardial injury and recovery after heart transplantation. Journal of Cardiothoracic Surgery 2013 8:165

\section{Submit your next manuscript to BioMed Central and take full advantage of:}

- Convenient online submission

- Thorough peer review

- No space constraints or color figure charges

- Immediate publication on acceptance

- Inclusion in PubMed, CAS, Scopus and Google Scholar

- Research which is freely available for redistribution 